\title{
nature methods
}

AMENDMENTS

\section{Author Correction: Deep learning improves macromolecule identification in 3D cellular cryo-electron tomograms}

Emmanuel Moebel, Antonio Martinez-Sanchez DD, Lorenz Lamm, Ricardo D. Righetto, Wojciech Wietrzynski, Sahradha Albert, Damien Larivière, Eric Fourmentin (D), Stefan Pfeffer (D), Julio Ortiz, Wolfgang Baumeister (D), Tingying Peng, Benjamin D. Engel (iD and Charles Kervrann (D)

Correction to: Nature Methods https://doi.org/10.1038/s41592-021-01275-4, published online 21 October 2021.

In the version of this Article initially published, there was an error in Fig. 2b. The image labeled "Segmentation target" was a duplicate of Fig. 2a; the image has been replaced with the correct version. In the Fig. 4 caption for panels "b,c, Score maps...," the text "( $25 \AA)$ " has been removed from the end of the sentence. For the final table in the online Methods, under "Evaluation," the data are unchanged but have been reorganized for clarity. Finally, the two callouts to "Fig. 4" in Extended Data Fig. 5 caption should instead have referred to "Extended Data Fig. 4" and have now been corrected.

The changes have been made to the online version of the article.

Published online: 16 November 2021

https://doi.org/10.1038/s41592-021-01349-3

(c) The Author(s), under exclusive licence to Springer Nature America, Inc. 2021

\section{Author Correction: A 4D single-cell protein atlas of transcription factors delineates spatiotemporal patterning during embryogenesis}

Xuehua Ma ID, Zhiguang Zhao, Long Xiao, Weina Xu, Yahui Kou, Yanping Zhang, Gang Wu, Yangyang Wang and Zhuo Du(iD

Correction to: Nature Methods https://doi.org/10.1038/s41592-021-01216-1, published online 26 July 2021.

In the version of this article initially published, a composition error appeared in two places. In the penultimate sentence in the third paragraph of the "A winding trajectory of cell state progression" subsection, in the text now reading "Moreover, undirectional transitions were widely observed in cells within clonal cell lineages that produced an identical cell type (Fig. 6e)," the word "undirectional" initially appeared as "unidirectional." The current Fig. 6 ("Systematic undirectional state transition during cell differentiation") was affected by the same error. The changes have been made to the HTML and PDF versions of the article.

Published online: 8 December 2021

https://oi.org/10.1038/s41592-021-01366-2

(c) The Author(s), under exclusive licence to Springer Nature America, Inc. 2021 